\title{
The Impact of Red Noise in Radial Velocity Planet Searches
}

\author{
Roman V. Baluev ${ }^{1,2}$ \\ ${ }^{1}$ Pulkovo Astronomical Observatory, \\ Pulkovskoje sh. 65/1, Saint Petersburg 196140, Russia \\ ${ }^{2}$ Sobolev Astronomical Institute, Saint Petersburg State University, \\ Universitetskij pr. 28, Petrodvorets, Saint Petersburg 198504, Russia \\ email: roman@astro.spbu.ru
}

\begin{abstract}
We demonstrate that moderate exoplanet radial velocity searches are often subject to the effect of the correlated (red) radial velocity noise. When disregarded, this effect may induce strong distortions in the results of the time series analysis and, ultimately, can even lead to false planet detections. We construct a maximum-likelihood algorithm, which is able to manage this issue rather efficiently.
\end{abstract}

Keywords. methods: data analysis, methods: statistical, techniques: radial velocities

Traditionally, when analysing radial velocity (RV) time series of exoplanetary surveys, we assume that these measurements possess mutually independent (uncorrelated) errors. Such uncorrelated noise is also called white, since its frequency spectrum is uniform. This is only an assumption, however: various activity effects can easily generate autocorrelated RV noise.

We find that the residual RV noise of many known planet-hosting stars demonstrate non-uniform frequency spectra (see Fig. 1 as example for GJ876). According to the Wiener-Khinchin theorem, such non-white spectra indicate autocorrelated noise. Obviously, this correlated structure affects the results of any data analysis, and should be taken into account.

We find that the shape of the residual power spectrum is well consistent with the socalled red-noise model: we can see a low-frequency band with excessive power (the red noise itself), its diurnal alias band around the frequency of $1 \mathrm{day}^{-1}$, and a depression in the middle.

The particular examples are the stars GJ876 (Baluev 2011) and GJ581, both are M dwarfs hosting multi-planet extrasolar systems. For these stars, the red noise is seen simultaneously in the time series issued by different observatories, confirming that its "redness" is caused by the star itself, rather than by the instruments.

Typically, the parametric uncertainties are underestimated, if we do not take the noise correlations into account. Also, the estimated values for some of the parameters may shift systematically. Ultimately, the non-white noise may result in false planet detections, which is probably the case of the putative planet candidates GJ581 $\mathrm{f}$ and g announced by Vogt et al. (2010). For this exo-system, the periodogram peaks previously interpreted as planets $\mathrm{f}$ and $\mathrm{g}$ disappear when we take the red noise into account.

We analysed RV data for the 23 HARPS planet-hosting targets currently available in the Vizier catalogue database. Four stars (GJ876, GJ581, HD145377, HD40307) demonstrated clearly non-uniform residual frequency spectra, implying that $\sim 20 \%$ of the stars generate this type of RV noise. However, if we select only the stars for which such 

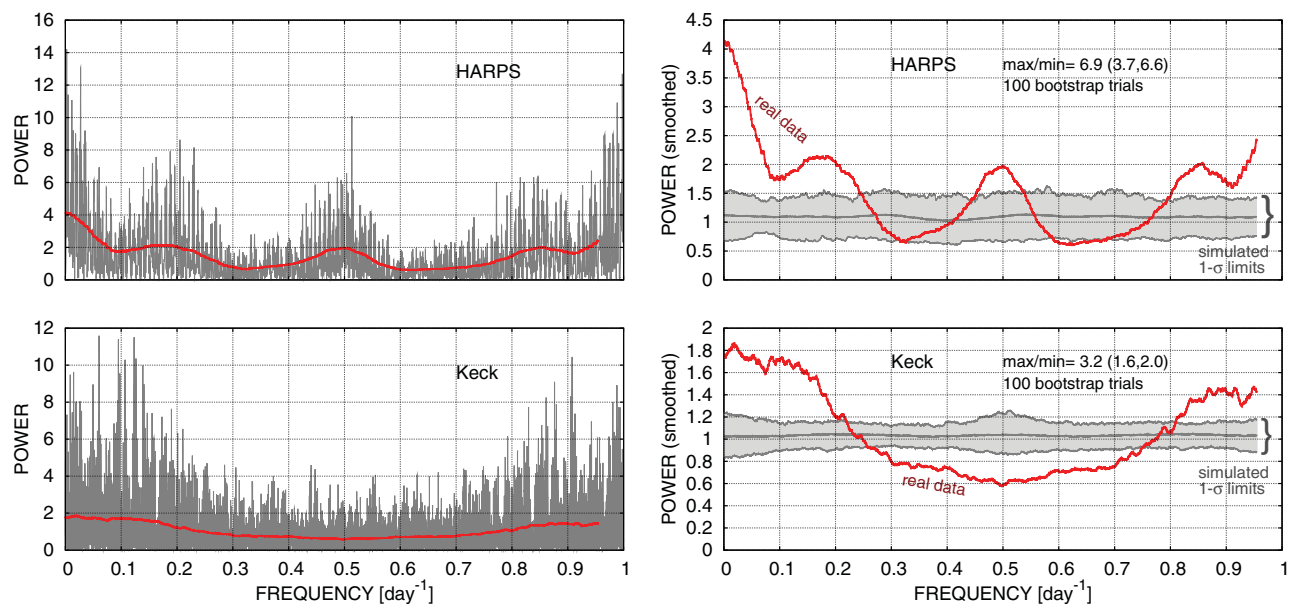

Figure 1. Raw (left) and smoothed (right) residual periodograms for RV data for the four-planet GJ876 extrasolar system. The contribution of the known four planets was subtracted off when constructing these periodograms. The periodograms show systematic deviation from the uniform (white) frequency spectrum: a prominence at low frequencies (long periods), accompanied by its alias at unit frequency (diurnal alias period), with a depression in the middle. These deviations are well above expected statistical limits for the white noise, and are statistically significant. See details in (Baluev 2011).

correlatedness is practically detectable, say only 15 targets for which we have at least 50 RV measurements, then the fraction of "red-noising" stars grows even more, to $\sim 25 \%$.

We provide a working maximum-likelihood data analysis algorithm to deal with this issue in the first approximation (Baluev 2011). However, this correlated noise requires a detailed investigation in the future, since we need to have a reliable error model for ongoing and future RV exoplanetary surveys.

This work was supported by the Russian Academy of Sciences (programme "Origin and Evolution of Stars and Galaxies") and by the Russian President programme of support of leading scientific schools (grant NSh.3290.2010.2).

\section{References}

Baluev, R. V. 2011, Celest. Mech. Dyn. Astron., 111, 235

Vogt, R. V. 2010, ApJ, 723, 954 